\title{
Sarcopenia and Myosteatosis as Prognostic Markers in Patients with Advanced Cholangiocarcinoma Undergoing Palliative Treatment
}

\author{
Markus S. Jördens ${ }^{1, *},+\left(\mathbb{D}\right.$, Linda Wittig ${ }^{1, \dagger}{ }^{,}$Lisa Heinrichs ${ }^{1} \oplus$, Verena Keitel ${ }^{1}$, Maximilian Schulze-Hagen ${ }^{2}{ }^{\oplus}$, \\ Gerald Antoch ${ }^{3}$, Wolfram T. Knoefel ${ }^{4}$, Georg Fluegen ${ }^{4}\left(\mathbb{D}\right.$, Tom Luedde ${ }^{1}$, Christina Loberg ${ }^{3, \neq}{ }^{10}$, \\ Christoph Roderburg ${ }^{1, \ddagger}$ and Sven H. Loosen ${ }^{1, *} \neq \pm$
}

\section{check for} updates

Citation: Jördens, M.S.; Wittig, L.; Heinrichs, L.; Keitel, V.; SchulzeHagen, M.; Antoch, G.; Knoefel, W.T.; Fluegen, G.; Luedde, T.; Loberg, C.; et al. Sarcopenia and Myosteatosis as Prognostic Markers in Patients with Advanced Cholangiocarcinoma Undergoing Palliative Treatment. J. Clin. Med. 2021, 10, 4340. https:// doi.org/10.3390/jcm10194340

Academic Editor: Jamie McPhee

Received: 3 September 2021

Accepted: 22 September 2021

Published: 23 September 2021

Publisher's Note: MDPI stays neutral with regard to jurisdictional claims in published maps and institutional affiliations.

Copyright: (c) 2021 by the authors. Licensee MDPI, Basel, Switzerland. This article is an open access article distributed under the terms and conditions of the Creative Commons Attribution (CC BY) license (https:/ / creativecommons.org/licenses/by/ $4.0 /)$.
1 Department of Gastroenterology, Hepatology and Infectious Diseases, University Hospital Düsseldorf, Medical Faculty of Heinrich Heine University Düsseldorf, 40225 Düsseldorf, Germany; linda.wittig@med.uni-duesseldorf.de (L.W.); lisa.heinrichs@med.uni-duesseldorf.de (L.H.); Verena.Keitel@med.uni-duesseldorf.de (V.K.); Tom.Luedde@med.uni-duesseldorf.de (T.L.); Christoph.Roderburg@med.uni-duesseldorf.de (C.R.)

2 Department for Diagnostic and Interventional Radiology, University Hospital Aachen, 52074 Aachen, Germany; mschulze@ukaachen.de

3 Department of Diagnostic and Interventional Radiology, University Hospital Düsseldorf, Medical Faculty of Heinrich Heine University Düsseldorf, 40225 Düsseldorf, Germany; gerald.antoch@med.uni-duesseldorf.de (G.A.); christina.loberg@med.uni-duesseldorf.de (C.L.)

4 Department of General, Visceral and Pediatric Surgery, University Hospital Düsseldorf, Medical Faculty of Heinrich Heine University Düsseldorf, 40225 Düsseldorf, Germany; knoefel@med.uni-duesseldorf.de (W.T.K.); Georg.Fluegen@med.uni-duesseldorf.de (G.F.)

* Correspondence: markus.joerdens@med.uni-duesseldorf.de (M.S.J.); Sven.Loosen@med.uni-duesseldorf.de (S.H.L.)

+ These authors share first authorship.

$\ddagger$ These authors share senior authorship.

Abstract: Background: Cholangiocarcinoma (CCA) represents the second most common primary liver cancer and is characterized by a very poor outcome, but reliable prognostic markers are largely missing. Sarcopenia, the progressive loss of muscle mass and strength, as well as myosteatosis have been associated with an unfavorable outcome in several clinical conditions, including cancer. Here, we evaluated the prognostic relevance of sarcopenia and myosteatosis using routine abdominal CT (computed tomography) scans in advanced stage CCA patients undergoing palliative treatment. Methods: Routine abdominal CT scans were used to assess the skeletal muscle and the psoas muscle index (L3SMI/L3PMI) at the level of the third lumbar vertebra as radiological indices for sarcopenia as well as the mean skeletal muscle attenuation (MMA) as a surrogate for myosteatosis. Results were correlated with clinical data and outcomes. Results: Using a calculated optimal cut-off value of $71.95 \mathrm{~mm}^{2} / \mathrm{cm}$, CCA patients with an L3SMI value below this cut-off showed a significantly reduced median overall survival (OS) of only 250 days compared to 450 days in patients with a higher L3SMI. Moreover, the median OS of CCA patients with an L3PMI above $6345 \mathrm{~mm}^{2} / \mathrm{cm}$ was 552 days compared to 252 days in patients with a lower L3PMI. Finally, CCA patients with an MMA above 30.51 Hounsfield Units survived significantly longer (median OS: 430 days) compared to patients with an MMA value below this ideal cut-off (median OS: 215 days). The prognostic relevance of L3SMI, L3PMI, and MMA was confirmed in uni- and multivariate Cox regression analyses. Conclusion: Routine abdominal CT scans represent a unique opportunity to evaluate sarcopenia as well as myosteatosis in advanced CCA patients. We identified the L3SMI/L3PMI as well as the MMA as negative prognostic factors in CCA patients undergoing palliative therapy, arguing that the "opportunistic" evaluation of these parameters might yield important clinical information in daily routine.

Keywords: cholangiocellular carcinoma; CCA; sarcopenia; myosteatosis; MMA; prognostic marker; survival 


\section{Introduction}

Cholangiocarcinoma (CCA) is the second most common primary malignancy of the liver and is characterized by a very poor outcome [1]. While surgical tumor resection represents a potentially curative therapeutic option in patients with early tumor stages, patients with locally advanced or metastatic CCA are usually attributed to a palliative treatment approach including systemic chemotherapy or active symptom control (ASC) in case of a poor clinical performance status [2]. The combination of gemcitabine and cisplatin has been established as the standard first-line therapy for unresectable CCA patients and significantly improves the median overall survival (OS) to 11.7 months [3]. Following progression to gemcitabine and cisplatin, the addition of FOLFOX (folinic acid, fluorouracil, and oxaliplatin) to ASC has been shown to improved median OS to 6.2 months compared to 5.3 months in the ASC-alone group [4]. However, treatment response and outcome to first and/or second-line chemotherapy is often heterogeneous, and prediction of the individual patients' prognosis has remained a challenge in clinical routine [5]. Especially in view of the fact that systemic therapy can be associated with significant side effects $[3,4]$ and predictive biomarkers are mainly experimental [6], the question arises as to which patients represent the ideal therapy candidates.

The term "sarcopenia" has recently been defined by the international working group on sarcopenia as the "progressive loss of muscle mass and strength with a risk of adverse outcomes such as disability, poor quality of life and death" [7]. In cancer patients, sarcopenia is most likely caused by systemic inflammatory processes that lead to catabolic nutrition state, a decreased appetite, as well as immobility [8], and it has previously been associated with an impaired outcome [9]. In addition, an increasing deterioration of muscle quality in the sense of myosteatosis is a frequently observed phenomenon in cancer patients, which is associated with an unfavorable prognosis [10]. As most cancer patients, including CCA patients, receive CT (computed tomography) scans to evaluate the tumor stage and response to therapy on a regular basis, these existing imaging data represent a unique opportunity to systematically assess sarcopenia and myosteatosis without further costs or burden on the patients.

Therefore, in the present study, we used routine CT scans to determine the skeletal muscle index (L3SMI) and the psoas muscle index (L3PMI) at the level of the third lumbar vertebra as radiological indices for sarcopenia as well as the mean skeletal muscle attenuation (MMA) as a surrogate for myosteatosis in order to evaluate its prognostic relevance in CCA patients undergoing palliative treatment.

\section{Patients and Methods}

\subsection{Selection of Study Patients}

We included 75 patients with CCA in palliative treatment in our study. All patients were treated at the Department of Gastroenterology, Hepatology and infectious diseases or the Department for General, Visceral, and Pediatric Surgery at the University Hospital Düsseldorf between 2011 and 2021 (Detailed patients' characteristics are presented in Table 1). In total, 64 patients underwent palliative systemic chemotherapy, and 11 patients received best supportive care. Fifty of the 75 patients died during the observation period, and 25 were lost to follow-up. Of all the included patients, we used CT scans performed before the start of chemotherapeutic treatment or best supportive care. Progressive disease was diagnosed by an experienced radiologist based on the radiological CT findings in restaging. In this context, the current RECIST criteria were applied. The study was approved by the ethics committee of the medical faculty of Heinrich Heine University Düsseldorf (2021-1334). 
Table 1. Study cohort.

\begin{tabular}{|c|c|}
\hline Parameter & Study Cohort \\
\hline CCA patients & $n=75$ \\
\hline \multicolumn{2}{|l|}{ Sex $\%(n)$} \\
\hline male & $53.3(40)$ \\
\hline female & $46.7(35)$ \\
\hline Age (years, median and range) & $70(30-87)$ \\
\hline \multicolumn{2}{|l|}{ BMI } \\
\hline Median $\mathrm{kg} / \mathrm{m}^{2}$ (range) & $24.2(18.5-44.3)$ \\
\hline \multicolumn{2}{|l|}{ BMI class $\mathrm{kg} / \mathrm{m}^{2} \%(n)$} \\
\hline BMI $<20$ & $9.3(7)$ \\
\hline BMI 20-25 & $45.3(34)$ \\
\hline BMI 25-30 & $28(21)$ \\
\hline $\mathrm{BMI}>30$ & $17.3(13)$ \\
\hline \multicolumn{2}{|l|}{ Systemic therapy \% $(n)$} \\
\hline Yes & $85.3(64)$ \\
\hline No & $14.7(11)$ \\
\hline \multicolumn{2}{|l|}{ Chemotherapy regimen $\%(n)$} \\
\hline Gemcitabine + Cisplatin & $81.3(52)$ \\
\hline Gemcitabine + Oxaliplatin & $4.7(3)$ \\
\hline Carboplatin + Paclitaxel & $1.6(1)$ \\
\hline Capecitabine Mono & $1.6(1)$ \\
\hline Gemcitabine Mono & $10.9(7)$ \\
\hline \multicolumn{2}{|l|}{ Tumor progression during follow-up? \% (n) } \\
\hline Yes & $28(21)$ \\
\hline No & $72(54)$ \\
\hline \multicolumn{2}{|l|}{ Metastatic Disease \% (n) } \\
\hline Yes & $69(52)$ \\
\hline No & $31(23)$ \\
\hline \multicolumn{2}{|l|}{ Localization of tumor metastasis \% $(n)$} \\
\hline Lymphatic & $20(15)$ \\
\hline Vascular & $8(6)$ \\
\hline Pulmonary & $18.7(14)$ \\
\hline Bone & $10.7(8)$ \\
\hline Suprarenal gland & $1.3(1)$ \\
\hline Peritoneum & $22.7(17)$ \\
\hline Other & $16(12)$ \\
\hline \multicolumn{2}{|l|}{ Pre-existing medical conditions \% (n) } \\
\hline Preceded tumor disease & $24(18)$ \\
\hline Preceded systemic chemotherapy & $1.3(1)$ \\
\hline Diabetes mell. Type 2 & $29.3(22)$ \\
\hline Arterial Hypertension & $58.7(44)$ \\
\hline Hepatitis B & $6.7(5)$ \\
\hline Hepatitis C & $5.3(4)$ \\
\hline Alcohol abuse & $1.3(1)$ \\
\hline Primary biliary cholangitis & $2.7(2)$ \\
\hline Primary sclerosing cholangitis & $1.3(1)$ \\
\hline Non-alcoholic steatohepatitis & $2.7(2)$ \\
\hline Inflammatory bowel disease & $1.3(1)$ \\
\hline Gastritis & $24(18)$ \\
\hline Overall survival (days, median and range) & $224(3-1059)$ \\
\hline Progression-free survival (days, median and range) & $132(3-916)$ \\
\hline
\end{tabular}
BMI, body mass index.

\subsection{Analysis of Sarcopenia}

We used the venous phase of CT scans obtained before the start of systemic therapy or best supportive care to analyze sarcopenia. For L3SMI, we measured the areas of the $\mathrm{m}$. psoas, $\mathrm{m}$. erector spinae, $\mathrm{m}$. quadratus lumborum, $\mathrm{m}$. rectus abdominis, $\mathrm{m}$. transversus abdominis, $\mathrm{m}$. obliquus abdominis internus, and $\mathrm{m}$. obliquus abdominis externus at the level of the third lumbar vertebra in a single slice with a semi-automatic segmentation tool 
(3D Slicer, [11]). To determine the psoas muscle index at the level of the 3rd lumbar vertebra (L3PMI), only the left m. psoas was measured (Figure 1). We quantified muscles with attenuation values between -29 and 150 Hounsfield Units (HU). The 3D Slicer software calculates the mean skeletal muscle attenuation (MMA) and the muscle area as an absolute value. MMA is defined as the mean muscle density, which directly correlates with the amount of fat deposition within the muscle. L3SMI is defined as the total muscle area at the level of the third lumbar vertebra normalized for patients ' height. The L3PMI is defined as the area of the left psoas muscle at the level of the third lumbar vertebra normalized for the patients' height [12].

A
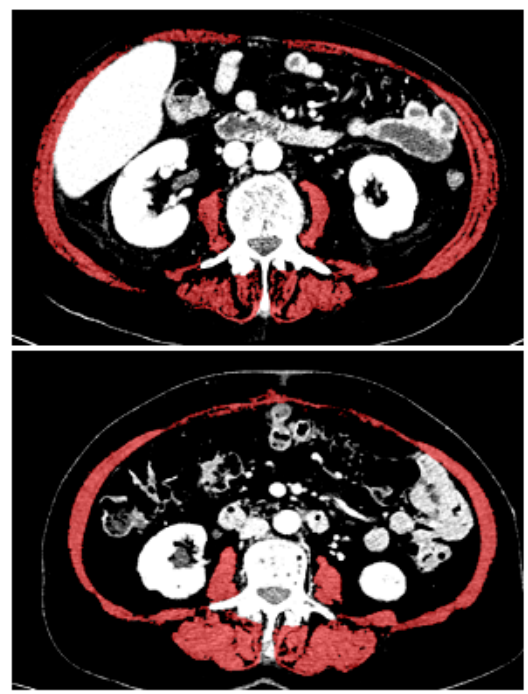

B
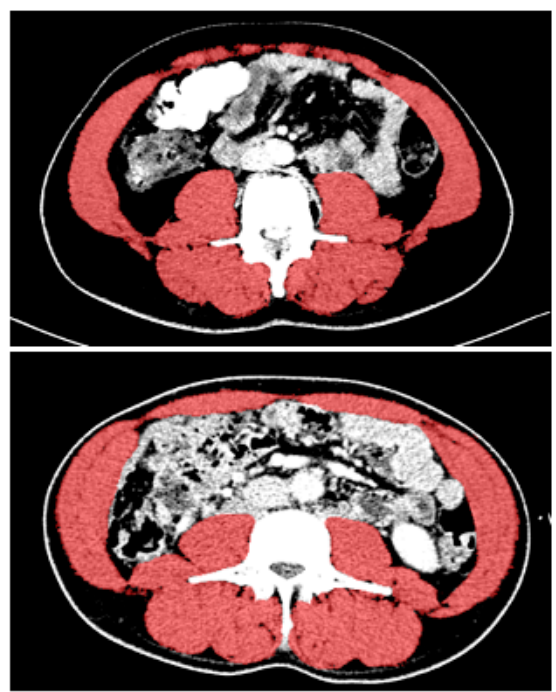

Figure 1. Evaluation of the L3SMI using the 3D slicer tool. Exemplary presentation of CT scans from patients with low (A) or high (B) L3SMI value.

\subsection{Statistical Analysis}

All statistical analyses were performed using SPSS 27 (SPSS, Chicago, IL, USA) unless otherwise described. Tests were similar performed as described in detail before [12]. To determine the normal distribution, a Shapiro-Wilk test was used. The Spearman correlation was performed for all correlation analyses. Using a Mann-Whitney U-test (two values) or Kruskal-Wallis test (more than two values), non-parametric data were compared. Medians, quartiles, and ranges are indicated in the shown box plots. Kaplan-Meier curves were generated to analyze the influence of different parameters on overall survival, and statistical differences between groups were tested with the log-rank test. To determine the optimal cut-off values for L3SMI, L3PMI, and MMA, we used the optimal cut-off finder, as described before [13]. Furthermore, we performed univariate and multivariate Cox regression analyses to analyze the prognostic value of different variables with regard to overall survival. The hazard ratio (HR) and 95\% confidence interval (CI 95\%) are displayed. $p$-values less than 0.05 are considered statistically significant.

\section{Results}

\subsection{Baseline Characteristics of Patients}

In this study 75 patients were included, of which $53.3 \%$ were male and $46.7 \%$ were female (Table 1). The median age was 70 years (range $30-87$ ), and the median BMI (body mass index) was 24.2 (18.5-44.3). Most (85.3\%) of the total patients had received palliative systemic chemotherapy as follows: Gemcitabine and Cisplatin $81.3 \%$, Gemcitabine and Oxaliplatin 4.7\%, Carboplatin and Paclitaxel 1.6\%, Capecitabine 1.6\%, Gemcitabine $10.7 \%$. More than one-quarter (28\%) of patients had progressive disease at 6 months follow up, while $69 \%$ had metastatic disease, and the localization of metastases was as follows: 
lymphatic $20 \%$, vascular infiltration $8 \%$, pulmonary $18.7 \%$, bone $10.7 \%$, suprarenal gland $1.3 \%$, peritoneal $22.7 \%$, other $16 \%$. Pre-existing medical conditions were as follows: preceded tumor disease $24 \%$, preceded systemic chemotherapy $1.3 \%$, diabetes mellitus type $229.3 \%$, arterial hypertension $58.7 \%$, hepatitis B $6.7 \%$, hepatitis C $5.3 \%$, abuse of alcohol $1.3 \%$, primary biliary cholangitis $2.7 \%$, primary sclerosing cholangitis $1.3 \%$, non-alcoholic steatohepatitis $2.7 \%$, inflammatory bowel disease $1.3 \%$, gastritis $24 \%$. The median overall survival of the study cohort was 224 (3-1059) days. Median progression-free survival was 132 (3-916) days. No difference between female or male sex regarding overall survival was identified ( $p=0.644$; Figure S1), and the presence of metastases had also no significant influence on overall survival ( $p=0.106$; Figure S2).

\subsection{L3SMI Is Dependent on Patients' Sex but Independent of Age, Albumin, or CRP Levels}

As expected, L3SMI before palliative therapy was higher in men compared to women $(p<0.001$; Figure 2A). There was no significant difference of the L3SMI between younger and older patients or between patients with a reduced or normal albumin serum concentration as a marker for malnutrition ( $p=0.551$ and $p=0.102$; Figure $2 \mathrm{~B}, \mathrm{C})$. To analyze the potential impact of inflammation on L3SMI, we compared patients with elevated CRP serum concentration with patients with normal CRP serum levels. However, CRP serum concentration had no effect on the L3SMI ( $p=0.664$; Figure 2D). We compared patients with normal or reduced hemoglobin serum levels as a marker for anemia and identified a clear trend toward a better L3SMI in non-anemic patients ( $p=0.074$; Figure 2E). Finally, we could not identify a significant difference of the L3SMI between patients with or without metastases as a marker for advanced disease stage ( $p=0.748$; Figure $2 \mathrm{~F})$.

A

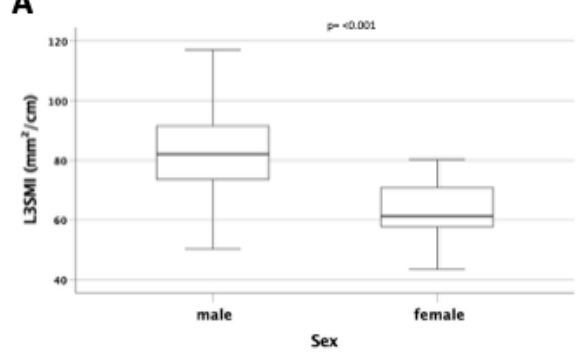

D

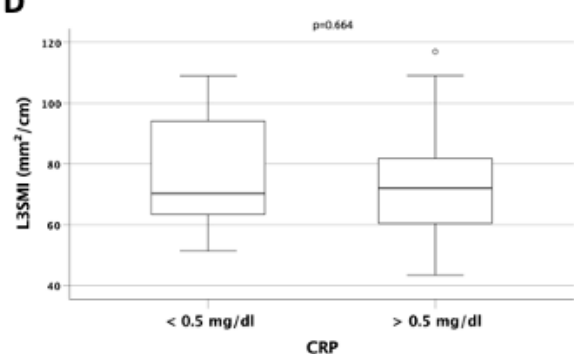

B

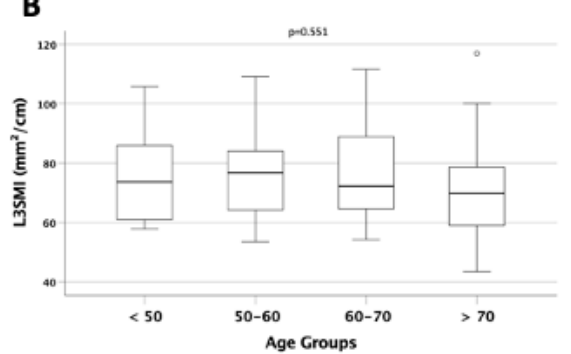

E

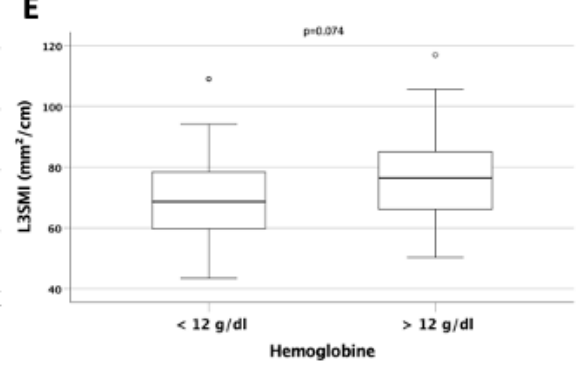

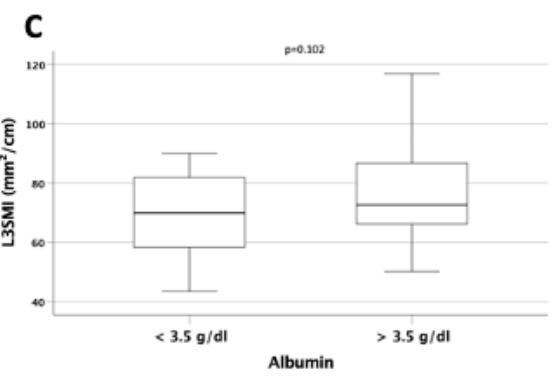

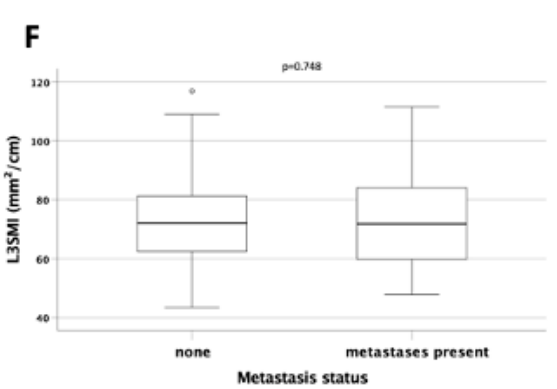

Figure 2. Evaluation of the L3SMI in different patient subgroups. (A) Male patients have a significantly higher L3SMI compared to female patients. The L3SMI is unaltered between patients of different age (B), with normal or reduced serum albumin levels (C) and normal or increased CRP serum levels (D). (E) Anemic patients show a trend toward a reduced L3SMI compared to non-anemic patients. The L3SMI is unaltered between patients with and without distant metastases (F).

\subsection{L3PMI Is Dependent on Sex and Albumin Serum Concentration but Independent of Age and $C P R$}

The L3PMI was also higher in male patients compared to female patients $(p<0.001$; Figure $3 \mathrm{~A}$ ), and there was no difference between different age groups or between patients with normal or elevated CRP serum concentrations ( $p=0.700$ and $p=0.885$; Figure 3B,D). In contrast to the L3SMI, we could identify a significantly higher L3PMI in patients with albumin serum concentrations above $3.5 \mathrm{~g} / \mathrm{dL}$ ( $p=0.049$; Figure 3C). Furthermore, neither 
serum hemoglobin concentration nor the presence of metastases had an influence on the L3PMI ( $p=0.136$ and $p=0.945$; Figure 3E,F).

A
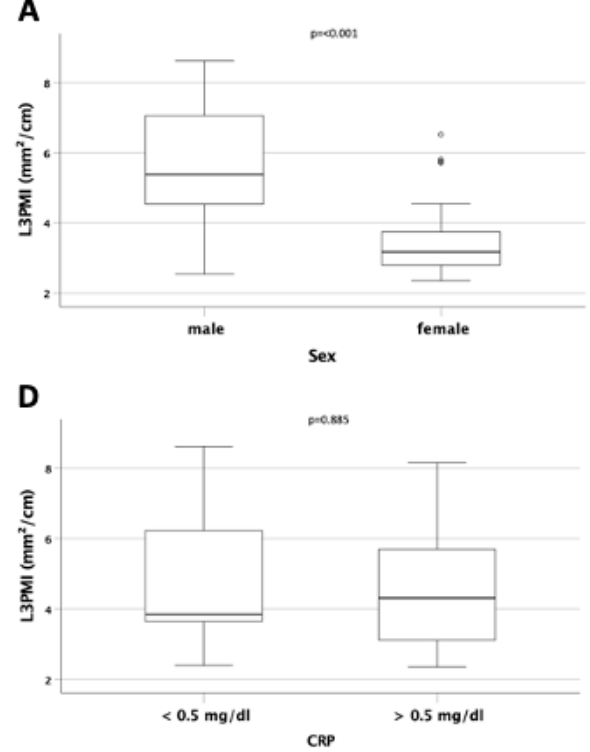

B

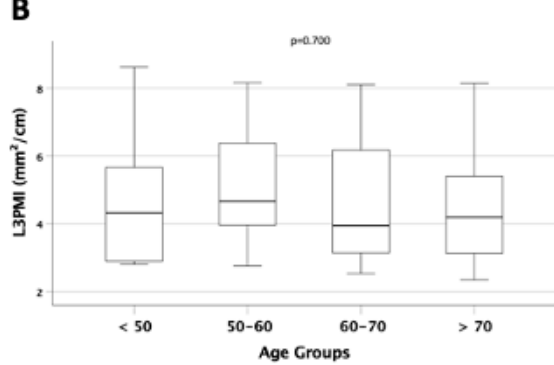

$\mathbf{E}$

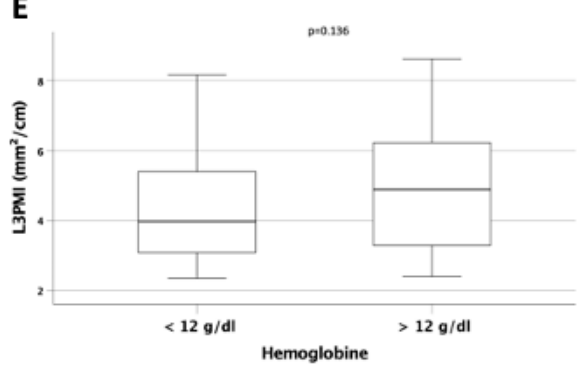

C

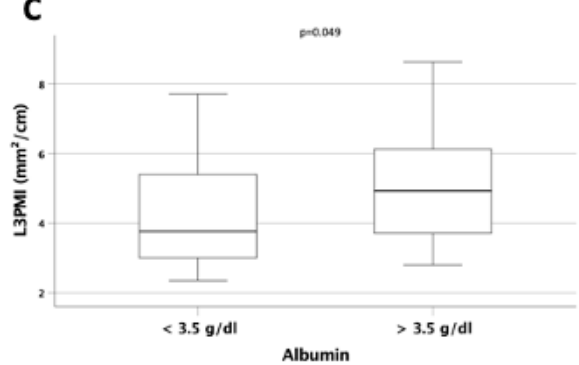

$\mathbf{F}$

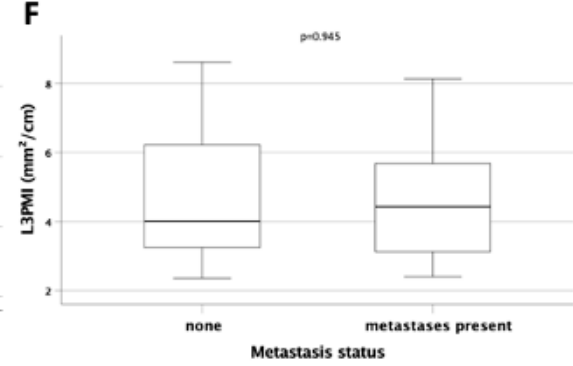

Figure 3. Evaluation of the L3PMI in different patient subgroups. (A) Male patients have a significantly higher L3PMI compared to female patients. The LPSMI is unaltered between patients of different age (B). (C) Patients with reduced serum albumin levels have a significantly lower LPSMI compared to patients with normal serum albumin levels. The L3PMI is unaltered between patients with normal or increased CRP serum levels (D), anemic or non-anemic patients (E), or patient with and without distant metastases (F).

\subsection{L3SMI and L3PMI Are Predictors of Overall Survival in CCA Patients under Palliative Therapy}

As sarcopenia is known to be a prognostic marker for overall survival (OS) in various malignant and non-malignant clinical conditions, we next evaluated a potential prognostic relevance of the L3SMI and L3PMI on patients' OS in our cohort of patients. We established ideal prognostic cut-off values using recently published software that identifies the respective L3SMI and L3PMI value with the most significant log-rank test in Kaplan-Meier curve analysis [13]. Using the optimal cut-off value, we could show that patients with an L3SMI value above $71.95 \mathrm{~mm}^{2} / \mathrm{cm}$ lived significantly longer compared to patient with an L3SMI below this value (430 days (95\%CI: $298-562$ ) vs. 250 days (95\%CI: 76-424) days; log rank $\chi^{2}(1)=5.622, p=0.18$; Figure 4A). In line, univariate Cox regression analysis revealed that an L3SMI value below the ideal cut-off value is a negative prognostic factor regarding OS (HR 1.990, 95\%CI: 1.115-3.551, $p=0.020$, Table 2). In line with these results, we could also establish a prognostic relevance of the L3PMI. When stratified according to the calculated ideal cut-off value of $6.345 \mathrm{~mm}^{2} / \mathrm{cm}$, the median OS of patients with an L3PMI above this value was significantly higher compared to patients with an L3PMI below (552 days (95\%CI: 413-690) vs. 252 days (95\%CI: 217-287); log rank $\chi^{2}(1)=4.600, p=0.32$; Figure 4B). Again, univariate Cox regression analysis revealed that an L3PMI value below the ideal cut-off value represents a negative prognostic factor for OS (HR 2.384, 95\%CI: 1.054-5.393, $p=0.037$, Table 2). 
A

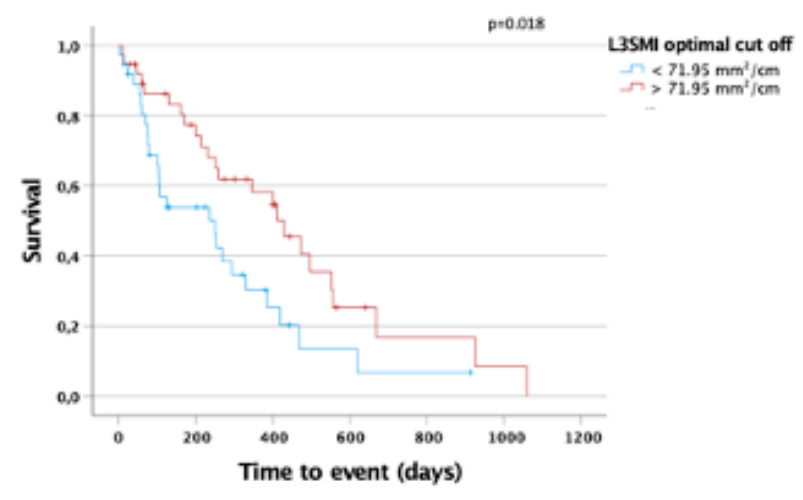

B

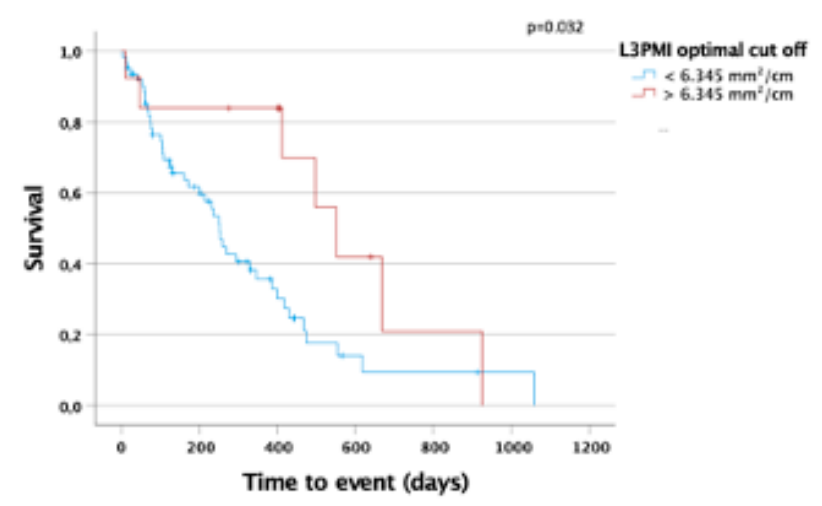

Figure 4. L3SMI and L3PMI are predictors of overall survival in CCA patients under palliative therapy. (A) CCA patients with an L3SMI value below the calculated ideal prognostic cut-off value of $71.95 \mathrm{~mm}^{2} / \mathrm{cm}$ show a significantly reduced overall survival compared to patients with an L3SMI above the cut-off. (B) CCA patients with an L3PMI value below the calculated ideal prognostic cut-off value of $6.345 \mathrm{~mm}^{2} / \mathrm{cm}$ show a significantly reduced overall survival compared to patients with an L3PMI above the cut-off.

Table 2. Univariate Cox regression analysis for the prediction of overall survival.

\begin{tabular}{|c|c|c|}
\hline Parameter & $p$-Value & Hazard Ratio (95\% CI) \\
\hline Sex & 0.645 & $1.145(0.644-2.037)$ \\
\hline Height & 0.205 & $0.978(0.945-1.012)$ \\
\hline Body weight & 0.225 & $0.988(0.969-1.008)$ \\
\hline BMI & 0.468 & $0.979(0.942-1.037)$ \\
\hline Age & 0.196 & $1.018(0.991-1.045)$ \\
\hline Preceded malignancy & 0.383 & $1.364(0.679-2.741)$ \\
\hline Diabetes & 0.785 & $1.092(0.582-2.049)$ \\
\hline Arterial hypertension & 0.381 & $0.774(0.435-1.374)$ \\
\hline Hepatitis B & 0.645 & $0.784(0.278-2.209)$ \\
\hline Hepatitis C & 0.085 & $0.402(0.143-1.133)$ \\
\hline Alcohol abuse & 0.971 & $0.963(0.132-7.051)$ \\
\hline PBC & 0.709 & $0.683(0.092-5.054)$ \\
\hline PSC & 0.564 & $1.800(0.244-13.299)$ \\
\hline CIBD & 0.582 & $0.571(0.078-4.199)$ \\
\hline Gastritis & 0.400 & $1.336(0.680-2.626)$ \\
\hline Lymphatic metastasis & 0.574 & $1.246(0.578-2.688)$ \\
\hline Vascular metastasis & 0.880 & $1.082(0.388-3.022)$ \\
\hline Pulmonary metastasis & 0.704 & $0.872(0.430-1.767)$ \\
\hline Osseus metastasis & 0.403 & $1.488(0.587-3.773)$ \\
\hline Suprarenal gland metastasis & 0.618 & $0.602(0.082-4.421)$ \\
\hline Peritoneal metastasis & 0.003 & $0.372(0.194-0.713)$ \\
\hline Other metastasis & 0.006 & $0.362(0.174-0.752)$ \\
\hline Any metastasis & 0.110 & $0.578(0.295-1.132)$ \\
\hline Sodium & 0.127 & $0.926(0.838-1.022)$ \\
\hline Potassium & 0.275 & $1.454(0.742-2.849)$ \\
\hline Calcium & 0.230 & $0.287(0.037-2.203)$ \\
\hline Creatinine & 0.649 & $0.979(0.895-1.071)$ \\
\hline GFR & 0.198 & $0.992(0.979-1.004)$ \\
\hline Uric acid & 0.784 & $1.033(0.818-1.305)$ \\
\hline Bilirubin & 0.318 & $1.066(0.940-1.209)$ \\
\hline ALT & 0.897 & $1.000(0.997-1.003)$ \\
\hline AST & 0.938 & $1.000(0.996-1.004)$ \\
\hline$\gamma \mathrm{GT}$ & 0.603 & $1.000(0.999-1.001)$ \\
\hline CRP & 0.002 & $1.124(1.044-1.211)$ \\
\hline Albumin & 0.207 & $0.646(0.328-1.274)$ \\
\hline Hemoglobin & 0.174 & $0.902(0.778-1.046)$ \\
\hline
\end{tabular}


Table 2. Cont.

\begin{tabular}{ccc}
\hline Parameter & $p$-Value & Hazard Ratio (95\% CI) \\
\hline MCV & 0.664 & $1.010(0.967-1.054)$ \\
MCH & 0.824 & $0.987(0.880-1.107)$ \\
Thrombocytes & $\mathbf{0 . 0 3 0}$ & $\mathbf{1 . 0 0 3 ( 1 . 0 0 0 - 1 . 0 0 6 )}$ \\
Quick & 0.605 & $1.006(0.983-1.029)$ \\
INR & 0.841 & $0.918(0.396-2.125)$ \\
aPTT & 0.754 & $1.009(0.955-1.066)$ \\
AFP & 0.861 & $1.000(1.000-1.000)$ \\
CEA & 0.174 & $1.001(0.999-1.004)$ \\
CA19-9 & 0.087 & $1.000(1.000-1.000)$ \\
L3SMI Cut-Off 71.95 & $\mathbf{0 . 0 2 0}$ & $\mathbf{1 . 9 9 0 ( 1 . 1 1 5 - 3 . 5 5 1 )}$ \\
L3PMI Cut-Off 6.345 & $\mathbf{0 . 0 3 7}$ & $\mathbf{2 . 3 8 4}(\mathbf{1 . 0 5 4 - 5 . 3 9 3 )}$ \\
MMA Cut-Off 30.51 & $\mathbf{0 . 0 1 1}$ & $\mathbf{2 . 1 7 6}(\mathbf{1 . 1 9 2}-\mathbf{3 . 9 7 1 )}$
\end{tabular}

BMI: Body Mass Index, PBC: primary biliary cholangitis, PSC: primary sclerotic cholangitis, CIBD: chronic inflammatory bowel disease, GFR: glomerular filtration rate, ALT: alanine aminotransferase, ALT: aspartate aminotransferase, $\gamma$ GT: Gamma-glutamyltransferase, CRP: C-reactive protein, MCV: mean corpuscular volume, $\mathrm{MCH}$ : mean corpuscular hemoglobin, INR: international normalized ratio, aPTT: activated partial thromboplastin time, AFP: alpha-fetoprotein, CEA: carcinoembryonic antigen, CA19-9: carbohydrate antigen 19-9, L3SMI: skeletal muscle index at level L3, L3PMI: psoas muscle index at L3, MMA: muscle mass attenuation. Bold denotes statistical significance $(p<0.05)$.

\subsection{The MMA Is a Predictor for Overall Survival in Patients with Palliative Treatment for CCA}

The mean skeletal muscle attenuation (MMA) represents a marker to assess the muscle's quality (myosteatosis) with lower values indicating a higher lipid concentration within the muscle [14]. We next calculated an optimal MMA cut-off value to evaluate a potential role of the MMA as a prognostic marker for OS in our cohort of patients. CCA patients with an MMA above 30.51 Hounsfield Units (HU) lived significantly longer compared to patients with an MMA value below this ideal cut-off (430 days ( $95 \% \mathrm{CI}$ : $234-626)$ vs. 215 days (95\%CI: 88-342); $\log \operatorname{rank} \chi^{2}(1)=6.709, p=0.010$; Figure 5A). To identify possible sex-specific differences for the MMA, we performed subgroup analyses and calculated sex-specific cut-off values for the MMA. Both in female $(28.54 \mathrm{HU})$ and male patients $(43.40 \mathrm{HU})$, the ideal sex-specific cut-off values showed a statistically significant prognostic value regarding median OS (female: 619 days (95\%CI: 189-1049) vs. 105 days (95\%CI: $57-153)$; $\log \operatorname{rank} \chi^{2}(1)=8.301, p=0.004$; male: 474 days (95\%CI: $\left.382-566\right)$ vs. 237 days (95\%CI: 188-286); log rank $\chi^{2}(1)=7.207, p=0.007$; Figure 5B,C). To further corroborate the prognostic relevance of the MMA, we performed uni- and multivariate Cox regression analyses. In univariate analysis, an MMA value below the ideal cut-off value represented a negative prognostic factor for OS (HR 2.384, 95\%CI: 1.054-5.393, $p=0.037$, Table 2). In multivariate analysis, the prognostic value of the MMA was independent of CRP, thrombocytes, routinely used tumor markers (CEA and CA19-9) as well as of the L3SMI and L3PMI (HR 2.264, 95\%CI: 1.059-4.842; $p=0.035$; Table 3).

A

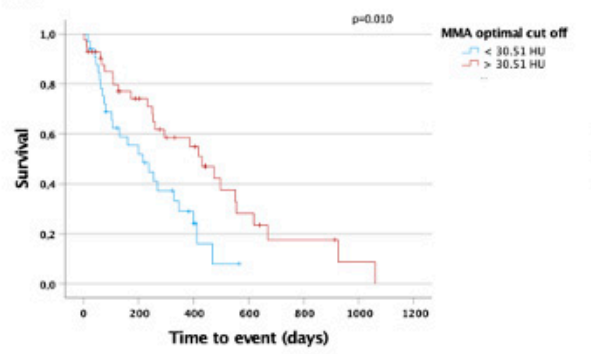

B

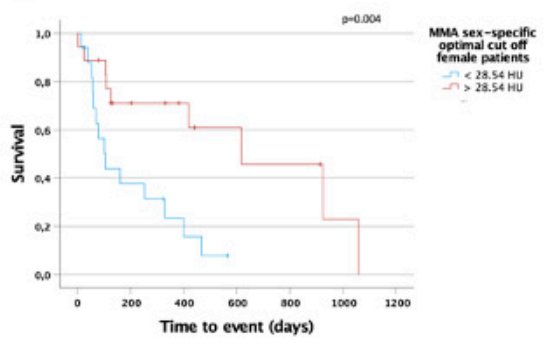

C

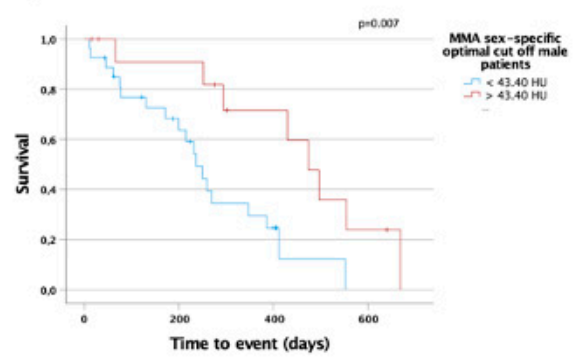

Figure 5. The MMA is a predictor for overall survival in patients with palliative treatment for CCA. (A) CCA patients with an MMA above 30.51 Hounsfield Units (HU) live significantly longer compared to patients with a MMA value below this ideal cut-off. Both in female ((B), 28.54 HU) and male patients $((\mathbf{C}), 43.40 \mathrm{HU})$, the ideal sex-specific cut-off values have a significant prognostic value regarding median OS. 
Table 3. Multivariate Cox regression analysis for the prediction of overall survival.

\begin{tabular}{ccc}
\hline Parameter & $p$-Value & Hazard Ratio (95\% CI) \\
\hline CRP & 0.462 & $1.060(0.908-1.236)$ \\
Thrombocytes & 0.101 & $1.003(0.999-1.007)$ \\
CEA & 0.846 & $1.000(0.997-1.003)$ \\
CA19-9 & 0.582 & $1.000(1.000-1.000)$ \\
L3SMI Cut-Off 71.95 & 0.748 & $0.854(0.326-2.237)$ \\
L3PMI Cut-Off 6.345 & 0.462 & $1.463(0.530-4.038)$ \\
MMA Cut-Off 30.51 & $\mathbf{0 . 0 3 5}$ & $\mathbf{2 . 2 6 4}(\mathbf{1 . 0 5 9 - 4 . 8 4 2 )}$
\end{tabular}

CRP: C-reactive protein, CEA: Carcinoembryonic antigen, CA19-9: Carbohydrate antigen 19-9, L3SMI: skeletal muscle index at level L3, L3PMI: psoas muscle index at L3, MMA: muscle mass attenuation. Bold denotes statistical significance $(p<0.05)$.

\subsection{The Combination of MMA and L3SMI Is a Strong Predictor for Overall Survival}

To further evaluate the prognostic power of muscle mass and quality, we performed Kaplan-Meier analyses and combined L3SMI as a marker for muscle mass and MMA as a marker for muscle quality. Patients with L3SMI and MMA above the before established cut-off values had a significantly better overall survival then patients with L3SMI and MMA under the optimal cut-off values. The overall survival of patients with either L3SMI or MMA over the optimal cut-off had a better survival than patients with both values under the optimal cut-off but worse than patients with both values over the optimal cut-off. This observation strengthens our assertion that both muscle mass and quality are important prognostic markers for overall survival in patients undergoing palliative therapy for CCA and that the combination of both is especially valuable.

\section{Discussion}

Different lines of evidence are available for a role of the body composition as a predictive and/or prognostic marker in patients with gastrointestinal cancers $[15,16]$. Both experimental and clinical analyses have demonstrated an impaired response to treatment and an impaired overall survival in patients with a reduced muscle mass and/or an impaired muscle quality [17]. Nevertheless, many questions including the magnitude of the associations and whether parameters that have been established in patients receiving surgery can be translated to patients in palliative disease stages have remained unanswered [18]. Here, we demonstrate that routine CT scans can be used to easily assess sarcopenia as well as myosteatosis in patients receiving palliative therapy for advancedstage CCA and that both parameters represent prognostic markers for OS. These findings suggest that "opportunistic" imaging analyses can yield important information on the individual patient's characteristics and should therefore be implemented into clinical routine.

The optimal method for body composition analysis has been the subject of intense debate in recent years. Many of the available methods can be biased, for example, by fluid overload, as it frequently occurs during the course of chemotherapy. Therefore, we used baseline CT scans to quantify the area of skeletal muscle as well as the skeletal muscle attenuation as a surrogate for sarcopenia. CT imaging is available for almost all CCA patients before chemotherapy, allowing cost-effective analyses of multiple surrogates for the body composition, as it was recently demonstrated in different clinical contexts [16,19]. Therefore, in contrast to most of the previous studies on biliary tract cancer that relied on measurements of the psoas muscle area only [20-22], we are able to provide a more complete picture of the individual body composition by simultaneously measuring muscle mass (L3SMI and L3PMI) and myosteatosis (MMA). So far, the role of sarcopenia and myosteatosis as prognostic factors in biliary tract cancer patients is largely unclear. Within this study, we quantified both the skeletal muscle mass and the amount of skeletal muscle fat deposition and demonstrated that an "impaired" body composition indicates an impaired clinical outcome in patients receiving chemotherapy for biliary tract cancer. At the respective optimal cut-off values that we established using a recently described biometric software [13], both a low L3SMI and L3PMI as well as a low skeletal muscle HU turned 
out to be powerful predictors of OS. Importantly, when both markers were combined (e.g., SMI low/MMA low vs. SMI high/MMA high patients), the prognostic potential was even further increased (see Figure 6).

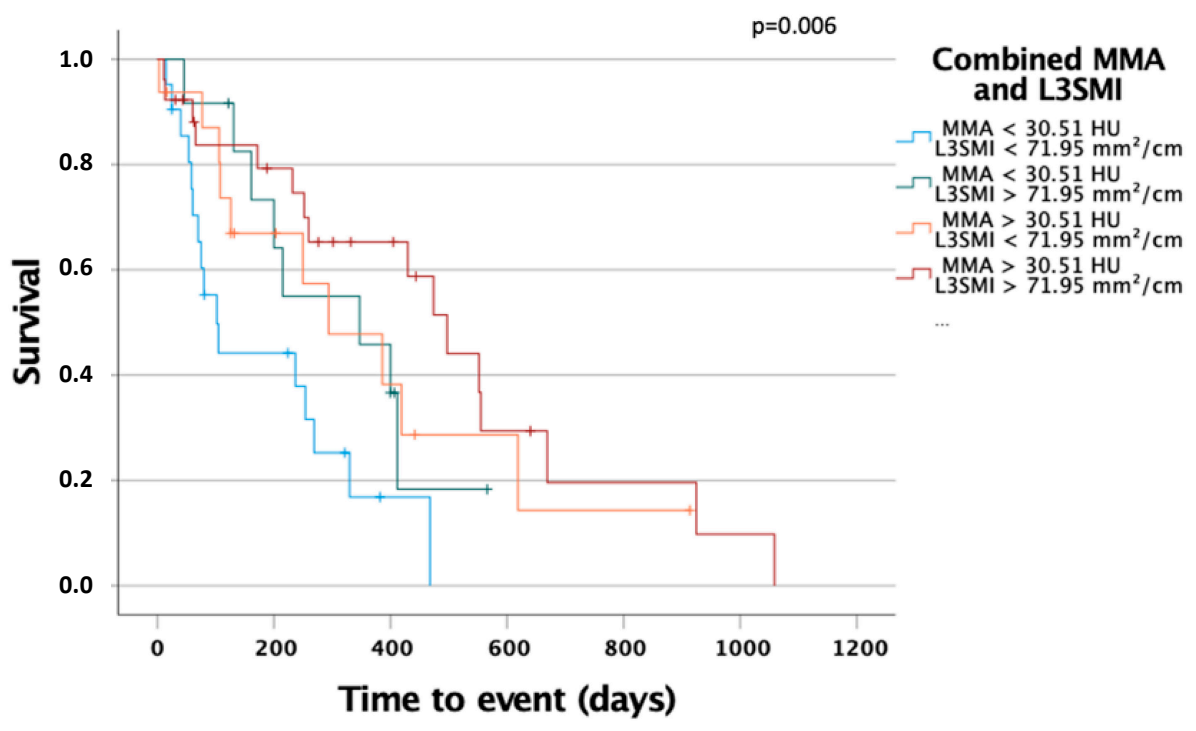

Figure 6. The combination of MMA and L3SMI is a strong predictor for overall survival in patients with palliative treatment for CCA. Patient with MMA above $30.51 \mathrm{HU}$ and L3SMI above $71.95 \mathrm{~mm}^{2} / \mathrm{cm}$ had significantly improved OS compared to patients with both markers under their optimal cut-off or combinations.

Along with these analyses suggesting a use of L3SMI, L3PMI, and MMA as stand-alone markers for estimating patients' overall survival, we further tested different combinations of L3SMI/MMA as prognostic markers, revealing that patients with low muscle mass and low muscle quality had the worst prognosis of all patients (Figure 6). Interestingly, these data are in line with a recent analysis by Yoon et al., demonstrating that both reduced muscle mass and muscle quality are indicative for an impaired prognosis of patients following the resection of biliary tract cancer [23]. In the past, reduced muscle mass, e.g., indicated by a reduced L3SMI or L3PMI, was used to assess the presence of sarcopenia. In patients with chronic diseases, low muscle mass is the result of an impaired muscle growth in the setting of an increased muscle wasting. However, it became clear that some patients show a much greater loss of muscle strength than would be expected after a loss of muscle mass. In these patients, increased intramuscular fat deposition has been described, as reflected by a change in MMA in our study [24]. In this context, a recently published study suggested that fatty degeneration of the muscle in tumor patients is an even earlier event; i.e., it occurs before loss of muscle mass [25]. Therefore, it seems likely that different pathophysiological mechanisms underlie these processes. Nevertheless, further clinical and translational studies are needed to fully understand the mechanism driving muscle loss and myosteatosis in cancer patients.

Sarcopenia is a common characteristic of elderly and moribund patients. Muscle wasting can be triggered by manifold disease conditions including cardiac failure and renal dysfunction [26]. As all of these factors are frequently found in patients with cancer and might themselves limit patients' prognosis, systematic research on the role of an impaired muscle mass and strength bears a risk of important bias. Nevertheless, in our analysis, we could identify L3SMI, L3PMI, and MMA as predictive factors for OS for patients undergoing palliative treatment for CCA. Especially MMA proved to be an independent and reliable prognostic factor regarding OS in multivariant regression analyses. Nevertheless, further studies are warranted to not only confirm their prognostic role in the context of biliary tract cancer but also to assess if the L3SMI, L3PMI, and the MMA could support future biomarker-driven clinical decision algorithms in the multimodal treatment of cancer. 
Importantly, our analysis only gave information on the prognosis of these patients but had no predictive value, meaning that it is unclear if those patients with an unfavorable prognosis in terms of their individual L3SMI, L3PMI, or MMA value might have had benefited to a greater extend from other treatments or even represent candidates for active symptom control who should not been administered chemotherapy. Moreover, we only evaluated SMI and MMA values before starting chemotherapy, but longitudinal measurements of SMI and MMA could also be important to answer the question of whether therapeutic interventions affecting body composition might influence patients' outcome. Furthermore, potential important information on factors influencing overall survival such as biliary stenting were often missing due to the nature of retrospective data and could not be analyzed. Nevertheless, our study demonstrated a potential prognostic value of sarcopenia and myosteatosis in the context of CCA patients. Moreover, our study demonstrated that the simultaneous assessment and combined analysis of different parameters of the patients individual body composition might be superior over a single marker for estimating the long-term outcome of these CCA patients. Such data might provide important information for clinical decision making on patients allocated to a systemic chemotherapy for CCA. Furthermore, these data can be used to decide on nutritional therapy in the context of multimodal treatment concepts in CCA patients. Therefore, our data should induce further clinical research but also basic research on the importance of body composition in the context of malignant diseases and CCA in particular.

Supplementary Materials: The following are available online at https:/ / www.mdpi.com/article/10 $.3390 /$ jcm10194340/s1, Figure S1: Overall survival is comparable between male and female CCA patients, Figure S2: Overall survival is comparable between CCA patients with or without distant metastases.

Author Contributions: Conceptualization, M.S.J., C.L., C.R. and S.H.L.; Formal analysis, L.W. and V.K.; Investigation, M.S.J. and L.W.; Methodology, M.S.J., L.W., L.H., M.S.-H., G.A., C.L. and S.H.L.; Project administration, C.R.; Resources, G.F. and T.L.; Supervision, W.T.K., C.L. and S.H.L.; Visualization, M.S.J.; Writing—original draft, M.S.J., L.W., C.R. and S.H.L.; Writing—review \& editing, M.S.J., L.H., V.K., M.S.-H., G.A., W.T.K., G.F., T.L., C.L., C.R. and S.H.L. All authors have read and agreed to the published version of the manuscript.

Funding: This research received no external funding.

Institutional Review Board Statement: The study was conducted according to the guidelines of the Declaration of Helsinki, and approved by the ethics committee of the medical faculty of Heinrich Heine University Düsseldorf (protocol code 2021-1334, date of approval 23 February 2021).

Informed Consent Statement: Patient consent was waived due to the pure retrospective data selection. This was approved by the ethics committee of the medical faculty of Heinrich Heine University Düsseldorf (2021-1334).

Data Availability Statement: Data are available upon request from the Department of Gastroenterology, Hepatology and Infectious Diseases of the University Hospital Düsseldorf for researchers who meet the criteria for access to confidential data: Wissenschaft.Gastro@med.uni-duesseldorf.de.

Conflicts of Interest: The authors declare no conflict of interest.

\section{References}

1. Razumilava, N.; Gores, G.J. Cholangiocarcinoma. Lancet 2014, 383, 2168-2179. [CrossRef]

2. Valle, J.W.; Kelley, R.K.; Nervi, B.; Oh, D.Y.; Zhu, A.X. Biliary tract cancer. Lancet 2021, 397, 428-444. [CrossRef]

3. Valle, J.; Wasan, H.; Palmer, D.H.; Cunningham, D.; Anthoney, A.; Maraveyas, A.; Madhusudan, S.; Iveson, T.; Hughes, S.; Pereira, S.P.; et al. Cisplatin plus Gemcitabine versus Gemcitabine for Biliary Tract Cancer. N. Engl. J. Med. 2010, 362, $1273-1281$. [CrossRef]

4. Lamarca, A.; Palmer, D.H.; Wasan, H.S.; Ross, P.J.; Ma, Y.T.; Arora, A.; Falk, S.; Gillmore, R.; Wadsley, J.; Patel, K.; et al. Second-line FOLFOX chemotherapy versus active symptom control for advanced biliary tract cancer (ABC-06): A phase 3, open-label, randomised, controlled trial. Lancet Oncol. 2021, 22, 690-701. [CrossRef]

5. Loosen, S.H.; Vucur, M.; Trautwein, C.; Roderburg, C.; Luedde, T. Circulating Biomarkers for Cholangiocarcinoma. Dig. Dis. 2018, 36, 281-288. [CrossRef] 
6. $\quad$ Macias, R.I.; Kornek, M.; Rodrigues, P.M.; Paiva, N.A.; Castro, R.E.; Urban, S.; Pereira, S.P.; Cadamuro, M.; Rupp, C.; Loosen, S.H.; et al. Diagnostic and prognostic biomarkers in cholangiocarcinoma. Liver Int. 2019, 39, 108-122. [CrossRef]

7. Fielding, R.A.; Vellas, B.; Evans, W.J.; Bhasin, S.; Morley, J.E.; Newman, A.B.; van Kan, G.A.; Andrieu, S.; Bauer, J.; Breuille, D.; et al. Sarcopenia: An Undiagnosed Condition in Older Adults. Current Consensus Definition: Prevalence, Etiology, and Consequences. International Working Group on Sarcopenia. J. Am. Med. Dir. Assoc. 2011, 12, 249-256. [CrossRef] [PubMed]

8. Arends, J.; Baracos, V.; Bertz, H.; Bozzetti, F.; Calder, P.C.; Deutz, N.E.; Erickson, N.; Laviano, A.; Lisanti, M.P.; Lobo, D.N.; et al. ESPEN expert group recommendations for action against cancer-related malnutrition. Clin. Nutr. 2017, 36, 1187-1196. [CrossRef] [PubMed]

9. Shachar, S.S.; Williams, G.R.; Muss, H.B.; Nishijima, T.F. Prognostic value of sarcopenia in adults with solid tumours: A meta-analysis and systematic review. Eur. J. Cancer 2016, 57, 58-67. [CrossRef] [PubMed]

10. Stretch, C.; Aubin, J.M.; Mickiewicz, B.; Leugner, D.; Al-Manasra, T.; Tobola, E.; Salazar, S.; Sutherland, F.R.; Ball, C.G.; Dixon, E.; et al. Sarcopenia and myosteatosis are accompanied by distinct biological profiles in patients with pancreatic and periampullary adenocarcinomas. PLoS ONE 2018, 13, e0196235. [CrossRef]

11. Fedorov, A.; Beichel, R.; Kalpathy-Cramer, J.; Finet, J.; Fillion-Robin, J.C.; Pujol, S.; Bauer, C.; Jennings, D.; Fennessy, F.; Sonka, M.; et al. 3D Slicer as an image computing platform for the Quantitative Imaging Network. Magn. Reson. Imaging 2012, 30, $1323-1341$. [CrossRef]

12. Loosen, S.H.; Bosch, V.V.; Gorgulho, J.; Schulze-Hagen, M.; Kandler, J.; Jördens, M.S.; Tacke, F.; Loberg, C.; Antoch, G.; Brümmendorf, T.; et al. Progressive Sarcopenia Correlates with Poor Response and Outcome to Immune Checkpoint Inhibitor Therapy. J. Clin. Med. 2021, 10, 1361. [CrossRef] [PubMed]

13. Budczies, J.; Klauschen, F.; Sinn B v Győrffy, B.; Schmitt, W.D.; Darb-Esfahani, S.; Denkert, C. Cutoff Finder: A comprehensive and straightforward Web application enabling rapid biomarker cutoff optimization. PLoS ONE 2012, 7, e51862. [CrossRef] [PubMed]

14. Goodpaster, B.H.; Carlson, C.L.; Visser, M.; Kelley, D.E.; Scherzinger, A.; Harris, T.B.; Stamm, E.; Newman, A.B. Attenuation of skeletal muscle and strength in the elderly: The health ABC study. J. Appl. Physiol. 2001, 90, 2157-2165. [CrossRef] [PubMed]

15. Chai, V.W.; Chia, M.; Cocco, A.; Bhamidipaty, M.; D'Souza, B. Sarcopenia is a strong predictive factor of clinical and oncological outcomes following curative colorectal cancer resection. ANZ J. Surg. 2021, 91, E292-E297. [CrossRef]

16. Murachi, Y.; Sakai, D.; Koseki, J.; Inagaki, C.; Nishida, N.; Yamaguchi, T.; Satoh, T. Impact of sarcopenia in patients with advanced or recurrent colorectal cancer treated with regorafenib. Int. J. Clin. Oncol. 2021, 26, 409-416. [CrossRef] [PubMed]

17. Kano, M.; Hihara, J.; Tokumoto, N.; Kohashi, T.; Hara, T.; Shimbara, K.; Takahashi, S. Association between skeletal muscle loss and the response to nivolumab immunotherapy in advanced gastric cancer patients. Int. J. Clin. Oncol. 2021, 26, 523-531. [CrossRef] [PubMed]

18. Runkel, M.; Diallo, T.D.; Lang, S.A.; Bamberg, F.; Benndorf, M.; Fichtner-Feigl, S. The Role of Visceral Obesity, Sarcopenia and Sarcopenic Obesity on Surgical Outcomes After Liver Resections for Colorectal Metastases. World J. Surg. 2021, 45, $2218-2226$. [CrossRef]

19. Wang, J.B.; Xue, Z.; Lu, J.; He, Q.L.; Zheng, Z.F.; Xu, B.B.; Xie, J.W.; Li, P.; Xu, Y.; Lin, J.X.; et al. Effect of sarcopenia on shortand long-term outcomes in patients with gastric neuroendocrine neoplasms after radical gastrectomy: Results from a large, two-institution series. BMC Cancer 2020, 20, 1002. [CrossRef]

20. Umetsu, S.; Wakiya, T.; Ishido, K.; Kudo, D.; Kimura, N.; Miura, T.; Toyoki, Y.; Hakamada, K. Effect of sarcopenia on the outcomes after pancreaticoduodenectomy for distal cholangiocarcinoma. ANZ J. Surg. 2018, 88, E654-E658. [CrossRef]

21. Otsuji, H.; Yokoyama, Y.; Ebata, T.; Igami, T.; Sugawara, G.; Mizuno, T.; Nagino, M. Preoperative sarcopenia negatively impacts postoperative outcomes following major hepatectomy with extrahepatic bile duct resection. World J. Surg. 2015, 39, 1494-1500. [CrossRef]

22. Okumura, S.; Kaido, T.; Hamaguchi, Y.; Fujimoto, Y.; Kobayashi, A.; Iida, T.; Yagi, S.; Taura, K.; Hatano, E.; Uemoto, S. Impact of the preoperative quantity and quality of skeletal muscle on outcomes after resection of extrahepatic biliary malignancies. Surgery 2016, 159, 821-833. [CrossRef] [PubMed]

23. Yoon, S.B.; Choi, M.H.; Song, M.; Lee, J.H.; Lee, I.S.; Lee, M.A.; Hong, T.H.; Jung, E.S.; Choi, M.G. Impact of preoperative body compositions on survival following resection of biliary tract cancer. J. Cachexia Sarcopenia Muscle 2019, 10, 794-802. [CrossRef] [PubMed]

24. Hunter, S.K. Sex differences in human fatigability: Mechanisms and insight to physiological responses. Acta Physiol. 2014, 210, 768-789. [CrossRef] [PubMed]

25. Hayashi, N.; Ando, Y.; Gyawali, B.; Shimokata, T.; Maeda, O.; Fukaya, M.; Goto, H.; Nagino, M.; Kodera, Y. Low skeletal muscle density is associated with poor survival in patients who receive chemotherapy for metastatic gastric cancer. Oncol. Rep. 2016, 35, 1727-1731. [CrossRef] [PubMed]

26. Schefold, J.C.; Bierbrauer, J.; Weber-Carstens, S. Intensive care unit-acquired weakness (ICUAW) and muscle wasting in critically ill patients with severe sepsis and septic shock. J. Cachexia Sarcopenia Muscle 2010, 1, 147-157. [CrossRef] 